Pacific Journal of Mathematic 


\title{
LOCALLY INVARIANT TOPOLOGIES ON FREE GROUPS
}

\author{
Sidney A. MorRis AND Peter Nickolas
}

In 1948, M. I. Graev proved that the free topological group on a completely regular Hausdorff space is Hausdorff, by showing that the free group admits a certain locally invariant Hausdorff group topology. In 1964, S. Świerczkowski gave a different proof, which also depends on the construction of a locally invariant topology. Yet another such construction follows from work of $K$. Bicknell and $\mathrm{S}$. A. Morris. Graev's topology has proved to be essential in the investigation of free products of topological groups; Swierczkowski's topology is the key to the work of W. Taylor on varieties and homotopy laws; and Bicknell and Morris extend results of Abels on norms on free topological groups. In this paper, the three topologies are investigated in detail. It is seen that the Graev topology contains the Swierczkowski topology, which in turn contains that of Bicknell and Morris. These containments are shown to be proper in general. It is known that the topology of the free topological group is in general finer than each of these three topologies.

Introduction. If $X$ is a completely regular Hausdorff space, let $F(X)$ denote the free group on the set $X$. Clearly the finest group topology on $F(X)$ which gives $X$ its original topology must make $F(X)$ the free topological group on $X$. Because of this, a number of authors have constructed Hausdorff group topologies on $F(X)$ as a means of proving that the free topological group is Hausdorff. Moreover, most other proofs of this fact are easily seen to contain implicitly the construction of some group topology on $F(X)$.

In this paper we shall examine and compare the topologizations of $F(X)$ arising from three such constructions.

The topologies we study will all have the additional property of local invariance; that is, they have bases at the identity of sets invariant under inner automorphisms, or, equivalently, they are defined by families of invariant pseudometrics. (A pseudometric $\rho$ on a group is (two-sided) invariant if $\rho(a x b, a y b)=\rho(x, y)$ for all group elements $a, b, x, y$.) Such topologies arise naturally in the present context since they necessarily make the group operations continuous. 
1. The topologies and their properties. We remind the reader that the Graev free topological group on a completely regular Hausdorff pointed space $X$ with basepoint $e$ is a topological group $F(X)$ which, algebraically, is a free group with $X \backslash\{e\}$ as a free basis, and is such that any continuous map from $X$ to a topological group, sending $e$ to the identity, extends uniquely to a continuous homomorphism on $F(X)$. The basepoint $e$ becomes the identity of $F(X)$, and the generating copy of $X$ has the topology of the original space. See [10] for a survey of the elementary theory of free topological groups.

In this paper we shall in fact use $F(X)$ to denote the underlying group of the free topological group - that is, the free group generated by the set $X \backslash\{e\}$, with $e$ the identity of $F(X)$.

We now define the first topology of interest.

Graev's construction: By a continuous pseudometric on $X$, we mean a pseudometric inducing a topology contained in the given topology on $X$.

For any continuous pseudometric $\rho$ on $X$, we define a pseudometric $\rho^{\prime}$ on $F(X)$ as follows. Let $w=\alpha_{1}^{\varepsilon_{1}} \cdots \alpha_{n}^{\varepsilon_{n}}$ be a (not necessarily reduced) word in symbols $\left\{\alpha_{i}\right\}$, with $\varepsilon_{i}= \pm 1, i=1, \cdots, n$. For $u, v \in F(X)$ we define

$$
\rho^{\prime}(u, v)=\inf \left\{\sum_{i=1}^{n} \rho\left(a_{i}, b_{i}\right)\right\},
$$

where $a_{i}, b_{i} \in X, i=1, \cdots, n$, are such that $a_{1}^{\varepsilon_{1}} \cdots a_{n}^{\varepsilon_{n}}=u$ and $b_{1}^{\varepsilon_{1}} \cdots$ $b_{n}^{\varepsilon_{n}}=v$, and the infimum is taken over all words $w$ and all possible choices of $\left\{a_{i}\right\}$ and $\left\{b_{i}\right\}$.

A definition of $\rho^{\prime}$ equivalent to ours was given by Graev [4], who showed that $\rho^{\prime}$ is an invariant pseudometric (and a metric if $\rho$ is), which coincides with $\rho$ on $X$. Denote by $\tau_{1}$ the topology induced on $F(X)$ by the extensions $\rho^{\prime}$ of all the continuous pseudometrics $\rho$ on $X$. The properties of $\rho^{\prime}$ and $\tau_{1}$ will be discussed fully later, but one straightforward fact will be noted now.

It is easily checked that two-sided invariance is equivalent to the requirement that

$$
\rho(a b, c d) \leqq \rho(a, c)+\rho(b, d)
$$

for all group elements $a, b, c, d$. From the definition of $\rho^{\prime}$ we have the following: 
Proposition 1. The pseudometric $\rho^{\prime}$ is the largest two-sided invariant extension of $\rho$, for any pseudometric $\rho$ on $X$, and $\tau_{1}$ is the finest locally invariant topology on $F(X)$ inducing the original topology on $X$.

Świerczkowski's construction: In [8], Świerczkowski defined a topology on general free algebras, giving an explicit construction of neighborhoods of points. His construction may be rephrased easily in terms of pseudometrics, giving rise, in the case of free groups, to the following definition.

Let $w=w\left(\alpha_{1}, \cdots, \alpha_{n}\right)$ be any word in the symbols $\alpha_{1}, \cdots, \alpha_{n}$. (Thus, each $\alpha_{i}$ may occur many times in the expression for $w$.) Given $\rho$ and $X$ as before, and $u, v \in F(X)$, we define

$$
\rho^{2}(u, v)=\inf \left\{\sum_{i=1}^{n} \rho\left(a_{i}, b_{i}\right)\right\},
$$

where again the infimum is taken over all words $w$ and all choices of $\left\{a_{i}\right\},\left\{b_{i}\right\}$ such that $w\left(a_{1}, \cdots, a_{n}\right)=u$ and $w\left(b_{1}, \cdots, b_{n}\right)=v$. Let $\tau_{2}$ be the topology defined by all the pseudometrics $\rho^{2}$.

We shall now discuss the properties of $\tau_{1}$ and $\tau_{2}$ in detail. Several of the arguments we shall use here have certain features in common, so we shall spend a little time isolating some of these first.

Let $w=\alpha_{1}^{\varepsilon_{1}} \cdots \alpha_{n}^{\varepsilon_{n}}$ be a word in symbols $\left\{\alpha_{i}\right\}$, with $\varepsilon_{i}= \pm 1, i=$ $1, \cdots, n$, and for $a_{i}, b_{i} \in X$ write $u$ and $v$ for the group elements represented by $a_{1}^{\varepsilon_{1}} \cdots a_{n}^{\varepsilon_{n}}$ and $b_{1}^{\varepsilon_{1}} \cdots b_{n}^{\varepsilon_{n}}$ respectively. We may select in the word $a_{1}^{\varepsilon_{1}} \cdots a_{n}^{\varepsilon_{n}}$ a cancellation order; that is, a sequence of steps of the form "delete $e$ " or "replace an adjacent $x$ and $x^{-1}$ by $e$ ", at the end of which the word representing $u$ is reduced. Each symbol $a_{i}^{\varepsilon_{i}}$ remaining after reduction will be called essential, and the others inessential. Note that the cancellation order may not be unique, and that the essential symbols $a_{i}^{\varepsilon_{i}}$ arising from different cancellation orders may occur at different values of the subscript $i$; though clearly the final string of essential symbols in the reduced word is uniquely determined. Of course, a given symbol may occur at one point as an essential symbol, and at another as an inessential one.

Let a cancellation order be fixed for both $u$ and $v$. Then for any essential symbol $s=a_{i}^{\varepsilon_{i}}$ of $u$ we may form a sequence of columns

$$
\left(\begin{array}{c}
s \\
t_{1}
\end{array}\right)\left(\begin{array}{c}
s_{1}^{-1} \\
t_{1}^{-1}
\end{array}\right)\left(\begin{array}{c}
s_{1} \\
t_{2}
\end{array}\right)\left(\begin{array}{c}
s_{2}^{-1} \\
t_{2}^{-1}
\end{array}\right) \cdots,
$$

where each pair $\left(s, t_{1}\right),\left(s_{1}^{-1}, t_{1}^{-1}\right), \cdots$ is one of $\left(a_{1}^{\varepsilon_{1}}, b_{1}^{s_{1}}\right),\left(a_{2}^{\varepsilon_{2}}, b_{2}^{\varepsilon_{2}}\right), \cdots$, 
where $t_{1} t_{1}^{-1}, t_{2} t_{2}^{-1}, \cdots$ and $s_{1}^{-1} s_{1}, s_{2}^{-1} s_{2}, \cdots$ are replaced by $e$ in the cancellation orders for $v$ and $u$ respectively, and where the sequence ends with the next occurrence of an essential symbol of $u$ or $v$, or of $e$. Such a sequence may be formed from each essential symbol of $u$, and then, similarly, from each essential symbol of $v$ : we observe that any two of these sequences are either disjoint or are identical except for the order in which their columns are displayed. (We may form similar sequences from the remaining inessential symbols, but these will be of no interest to us.)

Given any column sequence, its normalization will be the sequence of columns $C_{1} C_{2} \cdots C_{m}$ obtained by replacing each $x^{-1}, x \in X$, by $x$ in the original sequence. For each $i, 1 \leqq i \leqq m$, let $C_{i}(1)$ and $C_{i}(2)$ denote respectively the upper and lower entries of $C_{i}$. Then it is clear from its construction that the normalized sequence has a pairing, in that either

$$
\left\{\begin{aligned}
C_{1}(1)= & C_{2}(1), C_{3}(1)=C_{4}(1), \cdots \\
& C_{2}(2)=C_{3}(2), C_{4}(2)=C_{5}(2), \cdots
\end{aligned}\right.
$$

or

$$
\left\{\begin{array}{c}
C_{2}(1)=C_{3}(1), C_{4}(1)=C_{5}(1), \cdots \\
C_{1}(2)=C_{2}(2), C_{3}(2)=C_{4}(2) \cdots
\end{array}\right.
$$

There are exactly two entries left unpaired, namely, either $C_{1}(1)$ or $C_{1}(2)$, and either $C_{m}(1)$ or $C_{m}(2)$.

We may perform a process of column-deletion in a normalized sequence $C_{1} C_{2} \cdots C_{m}$, as follows. If $C_{i}=C_{j}$ for $i<j$, we delete columns $C_{i+1}, C_{i+2}, \cdots, C_{j}$ if $j-i$ is even, and we delete columns $C_{i}, C_{i+1}, \cdots, C_{j}$ if $j-i$ is odd. This procedure is carried out repeatedly (the steps chosen, of course, may not be unique) until we obtain a sequence, say $C_{i_{1}}, C_{i_{2}}, \cdots, C_{i_{k}}$, which has distinct columns. It is easily checked that $k$ and $m$ have the same parity, and that (if $k>0) C_{i_{1}} C_{i_{2}} \cdots C_{i_{k}}$ has a pairing like that of $C_{1} C_{2} \cdots C_{m}$, with its two unpaired elements being equal to those of $C_{1} C_{2} \cdots C_{m}$, and occurring in the same positions.

(For example, if $C_{1}(2)$ and $C_{m}(1)$ are unpaired, then so are $C_{i_{1}}(2)$ and $C_{i_{k}}(1)$, and $C_{1}(2)=C_{i_{1}}(2)$ and $C_{m}(1)-C_{i_{k}}(1)$.)

In [4], Graev used an argument based on the idea of partitioning into column sequences to prove that for any pair of representations $a_{1}^{\varepsilon_{1}} \cdots a_{n_{n}}^{\varepsilon_{n}}$ and $b_{1}^{\varepsilon_{1}} \cdots b_{n}^{\varepsilon_{n}}$ of $u, v \in F(X)$, there is another pair, $c_{1}^{\varepsilon_{1}} \cdots c_{n^{\varepsilon_{n}}}^{\varepsilon_{1}}$ and $d_{1}^{\varepsilon_{1}} \cdots d_{n^{n}}^{\varepsilon_{n}}$ respectively, with the properties: 
(1) $\left\{c_{1}, \cdots, c_{n}, d_{1}, \cdots, d_{n}\right\}$ are taken only from among $e$ and the letters occurring in the reduced forms of $u$ and $v$, and

(2) $\sum_{i=1}^{n} \rho\left(a_{i}, b_{i}\right) \geqq \sum_{i=1}^{n} \rho\left(c_{i}, d_{i}\right)$

(3) at most $p+q$ of the terms $\rho\left(c_{i}, d_{i}\right)$ are nonzero, where $u$ and $v$ have reduced lengths $p$ and $q$ respectively. It follows that the infimum $\rho^{\prime}(u, v)$ is actually attained for some pair of representations, and, using this, Graev proved that the restriction of $\rho^{\prime}$ to $X$ is $\rho$ (so that $\tau_{1}$ induces the original topology on $X$ ) and that $\tau_{1}$ is Hausdorff.

Our next result is that the infimum $\rho^{2}(u, v)$ need not be attained, but we shall go on to show that results parallel to those just mentioned for $\rho^{\prime}$ and $\tau_{1}$ still hold for $\rho^{2}$ and $\tau_{2}$. Indeed it will be observed that our proofs carry over routinely to the case of $\rho^{\prime}$ and $\tau_{1}$ also, so that Graev's result on the infimum may be dispensed with entirely.

Proposition 2. The infimum defining $\rho^{2}(u, v)$ need not be attained by any pair of representations of $u$ and $v$.

Proof. Take $X$ to be the plane with the point $a$ deleted, and choose $x, y \in X$ so that $x, y, e$ are the vertices of an equilateral triangle of side $l=\sqrt{3} \rho(e, a)$ and center $a$, where $\rho$ is the Euclidean metric on the plane. If $w(\alpha, \beta, \gamma)=\alpha \beta^{-1} \gamma \beta^{-1}$, the choices $(\alpha, \beta, \gamma)=$ $(x, e, y)$ and $(\alpha, \beta, \gamma)=(b, b, b)$ for any $b \in X$, yield representations of $x y$ and $e$, respectively, giving an associated sum of distances $\rho(x, b)+$ $\rho(y, b)+\rho(e, b)$. It is easy to see that this sum approaches $\sqrt{3 l}$ as $b$ approaches $a$, so that $\rho^{2}(x y, e) \leqq \sqrt{3} l$. We shall show that no pair of representations can achieve this value.

Suppose that $w\left(a_{1}, \cdots, a_{n}\right)=x y$ and $w\left(b_{1}, \cdots, b_{n}\right)=e$, for some $w$, and some $a_{i}, b_{i} \in X, i=1, \cdots, n$. As described above, select a cancellation order, essential symbols and a partitioning into column sequences of these representations. One may easily check that the sequences containing the essential occurrences of $x$ and $y$ must be disjoint, so that both end with an occurrence of $e$. Normalize both sequences.

Now perform the column deletion process on the sequence for $x$, writing the transformed sequence as $C_{1} C_{2} \cdots C_{p}$ and, noting that $C_{1}$ is of the form $\left(\begin{array}{l}x \\ u\end{array}\right)$ for some $u$, with $x$ unpaired. Suppose that the normalized sequence for $y$ is $D_{1} D_{2} \cdots D_{q}$, and let $i_{0}$ be the least $i, 1 \leqq i \leqq q$, for which $D_{i}$ equals some $C_{j}$, if such an $i$ exists. Say $D_{i_{0}}=C_{j_{0}}$, noting that the choice of $j_{0}$ is unique. Assume for concreteness that $i_{0}$ is even. 
Now transform $D_{1} \cdots D_{i_{0}}$ into a sequence $D_{i_{1}} \cdots D_{i_{k}}$ (for some $k$ ) by column deletions. (By our choice of $i_{0}, k \neq 0$.) Then $k$ is even, $D_{1}(1)=D_{i_{1}}(1)=y$, and if $D_{i_{0}}=\left(\begin{array}{c}s \\ t\end{array}\right)$ then $D_{i_{k}}=\left(\begin{array}{c}s \\ t^{\prime}\end{array}\right)$ for some $t^{\prime}$, with $y$ and $s$ the unpaired elements of both sequences.

Write $c_{j}=\rho\left(C_{j}(1), C_{j}(2)\right)$ and $d_{m}=\rho\left(D_{i_{m}}(1), D_{i_{m}}(2)\right)$, for $1 \leqq j \leqq \rho$ and $1 \leqq m \leqq k$. If $t \neq t^{\prime}$ (so that in fact none of $D_{i_{1}}, \cdots, D_{i_{k}}$ equals any of $C_{1}, \cdots, C_{p}$ ), then

$$
\begin{aligned}
& \sum_{i=1}^{n} \rho\left(a_{i}, b_{i}\right) \geqq \begin{cases}\sum_{j=1}^{j_{0}} c_{j}+\sum_{j=j_{0}+1}^{p} c_{j}+\sum_{m=1}^{k} d_{m}, & j_{0} \text { even } \\
\sum_{j=1}^{j_{0}-1} c_{j}+\sum_{j=j_{0}}^{p} c_{j}+\sum_{m=1}^{k} d_{m}, & j_{0} \text { odd }\end{cases} \\
& \geqq \rho(x, s)+\rho(s, e)+\rho(y, s) \text {. }
\end{aligned}
$$

If $t=t^{\prime}$, so that $D_{i_{k}}=D_{i_{0}}=C_{j_{0}}=\left(\begin{array}{c}s \\ t\end{array}\right)$, then

$$
\begin{aligned}
& \sum_{i=1}^{n} \rho\left(a_{i}, b_{i}\right) \geqq \begin{cases}\sum_{1}^{j_{0}-1} c_{i}+\sum_{j_{0}}^{p} c_{j}+\sum_{1}^{i_{k-1}} d_{m}, & j_{0} \quad \text { even } \\
\sum_{1}^{j_{0}} c_{j}+\sum_{j_{0}+1}^{p} c_{j}+\sum_{1}^{i_{k-1}} d_{m}, & j_{0} \quad \text { odd }\end{cases} \\
& \geqq \rho(x, t)+\rho(t, e)+\rho(y, t) .
\end{aligned}
$$

A similar argument applies when $i_{0}$ is odd, so that in all cases, $\sum \rho\left(a_{i}, b_{i}\right)$ is greater than or equal to an expression $\rho(x, b)+\rho(b, e)+$ $\rho(y, b)$, for some $b \in X$. Finally, if no integer $i_{0}$ exists, $\sum \rho\left(a_{i}, b_{i}\right)$ is easily seen to be at least $\rho(x, e)+\rho(y, e)$. Since the latter equals $2 l$, and any sum $\rho(x, b)+\rho(b, e)+\rho(y, b)$ is strictly larger than $\sqrt{3} l$ (for $b \neq a$ ), the result follows.

We remark further that for representations $w\left(a_{1}, \cdots, a_{n}\right)=x y$ and $w\left(b_{1}, \cdots, b_{n}\right)=e$ with $a_{i}, b_{i} \in\{x, y, e\}, i=1, \cdots, n, \sum_{i=1}^{n} \rho\left(a_{i}, b_{i}\right)$ must be at least $2 l$.

Proposition 3. Let $G$ be the subgroup of $F(X)$ generated by $\left\{x_{1}, \cdots, x_{k}\right\} \subseteq X$, and set $m$ equal to the minimum distance among $\left\{\rho\left(x_{i}, x_{j}\right): x_{i} \neq x_{j}\right\}$ and $\left\{\rho\left(x_{i}, e\right): x_{i} \neq e\right\}$. Then $\rho^{2}(w, e) \geqq m$ for any $w \in G, w \neq e$.

Proof. Let $w=y_{1}^{\varepsilon_{1}} \cdots y_{p}^{\varepsilon_{p}} \neq e$, in reduced form, with $y_{i} \in$ $\left\{x_{1}, \cdots, x_{k}\right\}, i=1, \cdots, p$, and let $w\left(a_{1}, \cdots, a_{n}\right)$ and $w\left(b_{1}, \cdots, b_{n}\right)$ be representations of $w$ and $e$ respectively. Select a cancellation order, essential symbols, and a column partitioning as usual.

If the column sequence for any $y_{i}^{\varepsilon_{i}}$ ends in $e$, a column deletion 
argument shows the distance sum from our representations to be at least $\rho\left(y_{i}, e\right)$, giving the desired conclusion. Similarly, $\rho^{2}(w, e) \geqq m$ if the normalized sequence for any $y_{i}^{\varepsilon_{i}}$ ends with any $y_{j}$ different from $y_{i}$. But this must happen for some $y_{i}^{\mathrm{e}_{i}}$, since if each normalized sequence begins and ends with the same essential symbol, we may replace all inessential symbols by $e$ (which cannot affect the reduced forms of $w\left(a_{1}, \cdots, a_{n}\right)$ and $\left.w\left(b_{1}, \cdots, b_{n}\right)\right)$, and we then see that $w\left(a_{1}, \cdots, a_{n}\right)$ reduces to $e$, contradicting $w \neq e$. Thus $\rho^{2}(w, e) \geqq m$ as required.

COROLlary. If $X$ is (completely regular) Hausdorff, so is $\left(F(X), \tau_{2}\right)$.

Proof. If $X$ is Hausdorff and $x_{1}, \cdots, x_{k}$ are distinct, there is a continuous pseudometric $\rho$ on $X$ for which $m \geqq 1$, and then $\rho^{2}(w, e) \geqq$ 1 for any nontrivial word $w$ in the symbols $\left\{x_{1}, \cdots, x_{k}\right\}$. Hence $\tau_{2}$ is Hausdorff.

We note the next two propositions without proof; the arguments needed are very much in the spirit of those already presented.

Proposition 4. For $x, y \in X, \rho^{2}(x, y)=\rho(x, y)$, and $\tau_{2}$ induces the original topology on $X$.

Proposition 5. If $x_{1}, x_{2}, \cdots, x_{n}$ are (not necessarily distinct) elements of $X$ different from $e$, then

$$
\rho^{\prime}\left(x_{1} x_{2} \cdots x_{n}, e\right)=\sum_{i=1}^{n} \rho\left(x_{i}, e\right) .
$$

Of interest in light of Proposition 2, and also of use later, is the following result and its corollary.

Proposition 6. Let $\rho$ be the usual metric on $X=[0,1]$, and let $u, v \in F(X)$. Given representations $w\left(a_{1}, \cdots, a_{n}\right)$ and $w\left(b_{1}, \cdots, b_{n}\right)$ of $u$ and $v\left(a_{i}, b_{i} \in X, i=1, \cdots, n\right)$, there is another pair of representations $w^{\prime}\left(a_{1}^{\prime}, \cdots, a_{m}^{\prime}\right), w^{\prime}\left(b_{1}^{\prime}, \cdots, b_{m}^{\prime}\right)$, with $a_{1}^{\prime}, \cdots, a_{m}^{\prime}, b_{1}^{\prime}, \cdots, b_{m}^{\prime}$ chosen from $e$ and the letters occurring in the reduced forms of $u$ and $v$, such that

$$
\sum_{i=1}^{m} \rho\left(a_{i}^{\prime}, b_{i}^{\prime}\right) \leqq \sum_{j=1}^{n} \rho\left(a_{j}, b_{j}\right)
$$

CoROLlary. The infimum $\rho^{2}(u, v)$ is achieved for some pair of representations $w\left(a_{1}, \cdots, a_{n}\right)$ and $w\left(b_{1}, \cdots, b_{n}\right)$ with each $a_{i}$ and $b_{i}$ 
chosen from $e$ and the letters occurring in the reduced forms of $u$ and $v$.

Proof of Proposition 6. Let $x_{1}<x_{2}<\cdots<x_{p}$ run through $e$ and the distinct letters occurring in the reduced forms of $u$ and $v$. If any $a_{i}$ or $b_{i}, i=1, \cdots, n$ is less than $x_{i}$, it does not occur in the reduced form of $u$ or $v$, and so we may replace it at each occurrence by $x_{1}$ without affecting the reduced forms of our representations. It is easy to see, moreover, that the distance sum from the altered representations is no larger than that from the original ones. Similarly replacing all $a_{i}$ and $b_{i}$ larger than $x_{p}$ by $x_{p}$, we may assume that $x_{1} \leqq a_{i}, b_{i} \leqq x_{p}$ for each $i$.

For any $a, b \in\left[x_{1}, x_{p}\right]$, let $d(a, b)$ be the number of integers $j$ for which $a<x_{j}<b$ or $b<x_{j}<a$. We shall show how to exchange our representations for ones in which $d\left(a_{i}, b_{i}\right)=0$ for each $i$.

Suppose that $d\left(a_{i}, b_{i}\right)>0$. If $a_{i}<b_{i}$, let $j$ be the least integer such that $a_{i}<x_{j}<b_{i}$, and if $b_{i}<a_{i}$ let $j$ be the greatest such that $b_{i}<x_{j}<a_{i}$.

Define

$$
\begin{aligned}
& w_{1}\left(\alpha_{1}, \cdots, \alpha_{i-1}, \alpha_{i+1}, \cdots, \alpha_{n}, \alpha, \beta, \gamma\right) \\
& \quad=w\left(\alpha_{1}, \cdots, \alpha_{i-1},\left(\alpha \beta^{-1} \gamma\right), \alpha_{i+1}, \cdots, \alpha_{n}\right) .
\end{aligned}
$$

Then

$$
w_{1}\left(a_{1}, \cdots, a_{i-1}, a_{i+1}, \cdots, a_{n}, a_{i}, b_{i}, b_{i}\right)
$$

and

$$
w_{1}\left(b_{1}, \cdots, b_{i-1}, b_{i+1}, \cdots, b_{n}, x_{j}, x_{j}, b_{i}\right)
$$

are clearly representations of $u$ and $v$ respectively, and their associated distance sum differs from the previous one only in that $\rho\left(a_{i}, b_{i}\right)$ has been replaced by $\rho\left(a_{i}, x_{j}\right)+\rho\left(x_{j}, b_{i}\right)+\rho\left(b_{i}, b_{i}\right)$. The latter, though, is equal to $\rho\left(a_{i}, b_{i}\right)$, so the distance sum is in fact unaltered. We observe that $d\left(a_{i}, x_{j}\right)=d\left(b_{i}, b_{i}\right)=0$, and that $d\left(x_{j}, b_{i}\right)=d\left(a_{i}, b_{i}\right)-$ 1 , so it follows that a repeated application of the above procedure will yield representations of $u$ and $v$, which we again write as $w\left(a_{1}, \cdots, a_{n}\right), w\left(b_{1}, \cdots, b_{n}\right)$, in which $d\left(a_{i}, b_{i}\right)=0$ for each $i$. That is, for each $i$ there is a $j, 1 \leqq j \leqq p-1$, such that $a_{i}, b_{i} \in\left[x_{j}, x_{j+1}\right]$.

Fix a $j, 1 \leqq j \leqq p-1$. For each $i$, write $C_{i}=\left[a_{i}, b_{i}\right]$ if $a_{i} \leqq b_{i}$ and $C_{i}=\left[b_{i}, a_{i}\right]$ if $b_{i} \leqq a_{i}$, and suppose without loss of generality that $\left\{i: C_{i} \subseteq\left[x_{j}, x_{j+1}\right]\right\}=\{1,2, \cdots, k\}$ for some $k$. For $t \in\left(x_{j}, x_{j+1}\right)$, with $t \neq a_{i}, b_{i}, i=1, \cdots, k$, define $B(t)$ to be the number of intervals $C_{i}, i=1, \cdots, k$, for which $t \in C_{i}$. Equivalently, $B(t)=\sum_{i=1}^{k} \chi_{C_{i}}(t)$, 
for all $t$ in the domain of $B$ (wher $\chi_{C}$ is the characteristic function of $C$ ). Then we have

$$
\begin{aligned}
\sum_{i=1}^{k} \rho\left(a_{i}, b_{i}\right) & =\sum\left|b_{i}-a_{i}\right| \\
& =\sum \int_{x_{j}}^{x_{j+1}} \chi_{C_{i}}(t) d t \\
& =\int_{x_{j}}^{x_{j+1}} B(t) d t .
\end{aligned}
$$

Now choose a $t_{0}$ for which $B\left(t_{0}\right)$ is minimal, and, in our representations, replace each $a_{i}$ in $\left[x_{j}, t_{0}\right)$ by $a_{i}^{\prime}=x_{j}$, and each $a_{i}$ in $\left(t_{0}, x_{j+1}\right]$ by $a_{i}^{\prime}=x_{j+1}$. Similarly, replace $b_{i} \in\left[x_{j}, t_{0}\right)$ by $b_{i}^{\prime}=x_{j}$, and $b_{i} \in\left(t_{0}, x_{j+1}\right]$ by $b_{i}^{\prime}=x_{j+1}$.

The transformed representations must still represent $u$ and $v$, since no symbol which has been (nontrivially) replaced occurs in the reduced forms of $u$ or $v$.

Denote the closed interval with endpoints $a_{i}^{\prime}, b_{i}^{\prime}$ by $C_{i}^{\prime}, i=1, \cdots$, $k$, and set $B^{\prime}(t)=\sum_{i=1}^{k} \chi_{C_{i}^{\prime}}(t), t \in\left(x_{j}, x_{j+1}\right)$. As above, $\sum_{i=1}^{k} \rho\left(a_{i}^{\prime}, b_{i}^{\prime}\right)=$ $\int_{x_{i}}^{x_{j+1}} B^{\prime}(t) d t$. But exactly $B\left(t_{0}\right)$ of the intervals $C_{i}^{\prime}$ are equal to $\left[x_{j}, x_{j+1}\right]$, while the rest are either $\left\{x_{j}\right\}$ or $\left\{x_{j+1}\right\}$, so that $B^{\prime}(t)=B\left(t_{0}\right)$ for all $t$.

Therefore

$$
\begin{aligned}
\sum_{i=1}^{k} \rho\left(a_{i}^{\prime}, b_{i}^{\prime}\right) & =\left(z_{j+1}-x_{j}\right) B\left(t_{0}\right) \\
& \leqq \int_{x_{j}}^{x_{j+1}} B(t) d t \\
& =\sum_{i=1}^{k} \rho\left(a_{i}, b_{i}\right) .
\end{aligned}
$$

Hence the distance sum from the transformed representations is no larger than that from the earlier ones, and, carrying out this process for each $j, 1 \leqq j \leqq p-1$, we obtain representations of the desired kind.

Proof of the corollary. We observe that any representations $w\left(a_{1}, \cdots, a_{n}\right)$ and $w\left(b_{1}, \cdots, b_{n}\right)$ of $u$ and $v$ may be replaced by others in which the pairs $\left\{\left(a_{i}, b_{i}\right)\right\}$ are distinct, and which give a sum of distances no greater than $\sum_{1}^{n} \rho\left(a_{i}, b_{i}\right)$. For, if $\left(a_{i}, b_{i}\right)=\left(a_{j}, b_{j}\right)$ for $i \neq j$, define

$$
w_{1}\left(\alpha_{1}, \cdots, \alpha_{j-1}, \alpha_{j+1}, \cdots, \alpha_{n}\right)=w\left(\alpha_{1}, \cdots, \alpha_{j-1}, \alpha_{i}, \alpha_{j+1}, \cdots, \alpha_{n}\right) ;
$$

then clearly $w_{1}\left(a_{1}, \cdots, a_{j-1}, a_{j+1}, \cdots, a_{n}\right)$ and $w_{1}\left(b_{1}, \cdots, b_{j-1}, b_{j+1}, \cdots, b_{n}\right)$ are again representations of $u$ and $v$, and the distance sum has been decreased by $\rho\left(a_{j}, b_{j}\right)$. This procedure may be repeated until all pairs $\left(a_{i}, b_{i}\right)$ remaining are distinct. 
From this observation, and Proposition 6, it follows easily that the infimum defining $\rho^{2}(u, v)$ may be taken over a finite set only, and the corollary follows.

Let $\tau_{0}$ denote the topology of the free topological group $F(X)$. The next proposition extends to $\tau_{1}$ and $\tau_{2}$ a result proved in [4] for $\tau_{0}$. The proofs proceed by a straightforward use of the definitions of $\rho^{\prime}$ and $\rho^{2}$, and will be omitted.

Proposition 7. The topologies $\tau_{0}, \tau_{1}$ and $\tau_{2}$ are independent of the choice of basepoint. More precisely, if $e_{1}, e_{2} \in X$, let $F\left(X, e_{1}\right)$, $F\left(X, e_{2}\right)$ be the groups obtained by selecting $e_{1}, e_{2}$ (respectively) as basepoint. If $\phi:\left(X, e_{1}\right) \rightarrow F\left(X, e_{2}\right)$ is the (basepoint-preserving) map defined by $\phi(x)=x e_{1}^{-1}, x \in X$, then the extension of $\phi$ to a homomorphism $\Phi: F\left(X, e_{1}\right) \rightarrow F\left(X, e_{2}\right)$ is a topological isomorphism with respect to $\tau_{0}, \tau_{1}$ or $\tau_{2}$.

We now define and discuss the third topology of interest.

The construction using the path-connected embedding: If $G$ is a group, let $G^{*}$ be the set of continuous-from-the-right step functions from the half-open interval $[0,1)$ into $G$; that is, functions $f$ for which there is a partition $0=a_{0}<a_{1}<\cdots<a_{n}=1$ of $[0,1]$ such that $f$ is constant on each $\left[a_{i}, a_{i+1}\right)$. Given an invariant pseudometric $\rho$ on $G$ we may define another such pseudometric $\rho^{*}$ on $G^{*}$ by

$$
\rho^{*}(f, g)=\int_{0}^{1} \rho(f(t), g(t)) d t, \quad f, g \in G^{*} .
$$

With the topology defined by $\rho^{*}, G^{*}$ becomes a path-connected topological group (see the more general Theorem 1 of [6]), and the map $i$ defined by $i(g)(t)=g, t \in[0,1), g \in G$, is an isometric isomorphism of $G$ into $G^{*}$. We define the path $P \equiv P(f, g)$ between $f$ and $g$ in $G^{*}$ by

$$
P(s)(t)= \begin{cases}g(t), & 0 \leqq t<s \\ f(t), & s \leqq t<1,\end{cases}
$$

for $t \in[0,1), s \in[0,1]$.

Now suppose that $S=\left\{s_{1}, s_{2}, \cdots, s_{n}\right\}$ is a finite ordered subset of $X$ containing $e$. Let $G(S)$ be the subgroup of $F(X)$ generated by $S$, let $d_{S}$ be the discrete metric on $G(S)$ (that is, all nonzero distances are equal to 1 ), and let $d_{S}^{*}$ be the extension of $d_{S}$ to $G(S)^{*}$. Clearly $\bigcup_{j=1}^{n-1} P\left(i\left(s_{j}\right), i\left(s_{j+1}\right)\right)$ is homeomorphic to $[0,1]$, so by the complete regularity of $X$ there is a continuous map $\phi: X \rightarrow \bigcup_{j=1}^{n-1} P\left(i\left(s_{j}\right), i\left(s_{j+1}\right)\right)$ such that $\phi\left(s_{j}\right)=i\left(s_{j}\right), j=1, \cdots, n$. Extending $\phi$ to a continuous 
homomorphism $\Phi: F(X) \rightarrow G(S)^{*}$, where $F(X)$ is now the free topological group, we define a continuous invariant pseudometric $\rho_{S, \phi}$ on $F(X)$ by

$$
\rho_{S, \phi}(u, v)=d_{S}^{*}(\Phi(u), \Phi(v)), \quad u, v \in F(X) .
$$

(Note that $\rho_{S, \phi}$ is invariant since $d_{S}$ and $d_{S}^{*}$ are.)

A proof that the free topological group is Hausdorff was given in [2] using path-connected embedding, although no explicit construction was given there of a topology on the free group. The topology $\tau_{3}$ generated by all the pseudometrics $\rho_{S, \phi}$, for all choices of $S$, and all choices of $\phi$ of the kind indicated, may be seen as an expression in terms of invariant pseudometrics of the proof of [2].

Proposition 8. If $X$ is completely regular and Hausdorff, then $\tau_{3}$ is Hausdorff, and $X$ has its original topology and is a closed subspace of $\left(F(X), \tau_{3}\right)$.

Proof. If $w$ is any nontrivial word in letters from $S=$ $\left\{x_{1}, \cdots, x_{n}, e\right\} \subseteq X$, we form the discrete metric $d_{S}$ on $G(S)$ and its extension $d_{S}^{*}$ to $G(S)^{*}$. Let $\phi: X \rightarrow \bigcup_{i=1}^{n-1} P\left(i\left(x_{j}\right), i\left(x_{j+1}\right)\right) \cup P\left(i\left(x_{n}\right), i(e)\right) \subseteq$ $G(S)^{*}$ be any continuous map such that $\phi\left(x_{j}\right)=i\left(x_{j}\right), j=1, \cdots, n$, and extend $\phi$ to a homomorphism $\Phi: F(X) \rightarrow G(S)^{*}$. Then clearly $\Phi(w)(t)=w$ for all $t \in[0,1)$, so $\rho_{S, \phi}(w, e)=1$, and $\tau_{3}$ is Hausdorff. Indeed we see further that if $u \notin X, \rho_{S, \phi}(x, u)=1$ for each $x \in X$, and this shows that $X$ is closed in $\tau_{3}$.

By construction, each $\rho_{S, \phi}$ is continuous on $X$, in its original topology, so the latter contains the restriction of $\tau_{3}$ to $X$. On the other hand, let $U \neq X$ be open in the original topology, and let $x$ be any point in $U$. If $e \in U$, select any $y \in X \backslash U$, set $T=\{x, y, e\}$, and form $d_{T}, d_{T}^{*}$ and $\rho_{T, \psi}$, where $\psi: X \rightarrow G(T)^{*}$ satisfies $\psi(x)=i(x), \psi(e)=$ $i(e)$ and $\psi(X \backslash U)=\{i(y)\}$, and has extension $\Psi$ on $F(X)$. If $B=$ $\left\{w \in F(X): \rho_{T, w}(w, x)<1\right\}$, then $B \cap X$ is open in the restriction of $\tau_{3}$ to $X$. But for $z \in B \cap X$ we have

$$
\begin{aligned}
1>\rho_{T, \psi}(z, x) & =d_{T}^{*}(\Psi(z), \Psi(x)) \\
& =\int_{0}^{1} d_{T}(\Psi(z)(t), x) d t,
\end{aligned}
$$

so that for some $t \in[0,1), \Psi(z)(t)=x$, implying that $z \in U$. That is, $x \in B \cap X \subset U$, and $U$ is open in the restriction of $\tau_{3}$ to $X$. (Note that this argument works even when $x=e$.) A very similar argument gives the same conclusion in the case when $e \notin U$, and the equality of the two topologies follows. 
REMARK. We have not included proofs that $X$ is closed in $\tau_{1}$ and $\tau_{2}$. This may be proved directly, but will in fact follow from Proposition 8 and Theorem 1.

\section{The relations between the topologies.}

THEOREM 1. If $X$ is completely regular and Hausdorff, then

$$
\tau_{0} \supseteqq \tau_{1} \supseteqq \tau_{2} \supseteqq \tau_{3} \text {. }
$$

THEOREM 2. The above inclusions are in general strict. Specifically, if $\tau_{0}=\tau_{1}$, or if $\tau_{1}=\tau_{2}$, then $X$ has the property that any countable union of its closed subsets must be closed. When $X=$ $[0,1], \tau_{2} \neq \tau_{3}$.

Proof of Theorem 1. Of course $\tau_{0}$ is the strongest group topology on $F(X)$ inducing the original topology on $X$, and so $\tau_{1} \subseteq \tau_{0}$. The inclusion $\tau_{2} \subseteq \tau_{1}$ follows from Proposition 1 .

To show that $\tau_{3} \leqq \tau_{2}$, fix a finite set $S \subseteq X$ containing $e$, and define $d_{S}^{*}$ and $\rho_{S, \phi}$ as earlier, for any choice of $\phi$. If we let $\rho$ be the restriction of $\rho_{S, \phi}$ to $X, \rho$ is a continuous pseudometric on $X$, and we shall show that $\rho^{2} \geqq \rho_{S, \phi}$.

Suppose that for $\delta>0, \rho^{2}(u, v)<\delta$, for some $u, v \in F(X)$. Then there is a word $w\left(\alpha_{1}, \cdots, \alpha_{n}\right)$ and $a_{1}, \cdots, a_{n}, b_{1}, \cdots, b_{n} \in X$ such that $w\left(a_{1}, \cdots, a_{n}\right)=u, w\left(b_{1}, \cdots, b_{n}\right)=v$, and such that $\sum_{i=1}^{n} \delta_{i}<\delta$, where

$$
\delta_{i}=\rho\left(a_{i}, b_{i}\right)=\int_{0}^{1} d_{S}\left(\phi\left(a_{i}\right)(t), \phi\left(b_{i}\right)(t)\right) d t .
$$

Since $d_{S}$ is the discrete metric we have $\phi\left(a_{i}\right)(t)=\phi\left(b_{i}\right)(t)$ for $t$ in a set $A_{i}$ of measure $1-\delta_{i}$. Suppose that $w\left(\alpha_{1}, \cdots, \alpha_{n}\right)=\alpha_{i_{1}}^{\varepsilon_{1}} \cdots \alpha_{i_{k}}^{\varepsilon_{k}}$, a (not necessarily reduced) word in the symbols $\left\{\alpha_{1}, \cdots, \alpha_{n}\right\}$, with each $\varepsilon_{i}$ equal to \pm 1 . For each $l, 1 \leqq l \leqq k$, the pair $\left(a_{i_{l}}, b_{i_{l}}\right)$ is one of the pairs $\left(a_{1}, b_{1}\right), \cdots,\left(a_{n}, b_{n}\right)$, and so $\phi\left(a_{i_{l}}\right)(t)=\phi\left(b_{i_{l}}\right)(t)$ for $t$ in one of the sets $A_{1}, \cdots, A_{n}$.

Hence $\Phi(u)(t)=\phi\left(a_{i_{1}}\right)(t)^{\varepsilon_{1}} \cdots \phi\left(a_{i_{k}}\right)(t)^{\varepsilon_{k}}$ is equal to $\Phi(v)(t)=$ $\phi\left(b_{i_{1}}\right)(t)^{\varepsilon_{1}} \cdots \phi\left(b_{i_{k}}\right)(t)^{\varepsilon_{k}}$ for $t \in A=\bigcap_{i=1}^{n} A_{i}$, and the measure of $A$ is at least $1-\sum \delta_{i}$, which is larger than $1-\delta$. Therefore $\rho_{S, \phi}(u, v)=$ $\int_{0}^{1} d_{S}(\Phi(u)(t), \Phi(v)(t)) d t<\delta$, and $\rho_{S, \phi} \leqq \rho^{2}$ as required, proving the theorem.

\section{Proof of Theorem 2.}

Case 1: $\tau_{0}$ and $\tau_{1}$. The result stated here will appear with proof in [5]. See [3] and [7] for related results. 
Case 2: $\tau_{1}$ and $\tau_{2}$. The following result will be needed.

LEMma. Suppose that $\left\{A_{n}\right\}$ is a sequence of closed sets in $X$ and $e \notin \cup A_{n}$. If $\left\{k_{n}\right\}$ is an increasing sequence of positive integers for which $\sum\left(1 / k_{n}\right)$ converges, and if $B=\cup B_{n}=\cup\left(A_{n}\right)^{k_{n}}$, then $e \notin \bar{B}$, the closure of $B$ in $\tau_{1}$.

Proof. Since, for each $n, A_{n}$ is closed and $e \notin A_{n}$, there are continuous pseudometrics $\rho_{n}$ on $X$ such that $\rho_{n}\left(e, A_{n}\right) \geqq 1, n \in N$. If we define

$$
\rho(x, y)=\sum_{n=1}^{\infty} \min \left\{\rho_{n}(x, y),\left(k_{n}\right)^{-1}\right\}, \quad x, y \in X,
$$

we see that $\rho$ is again a continuous pseudometric on $X$ and that $\rho\left(e, A_{n}\right) \geqq\left(k_{n}\right)^{-1}, n \in N$. Then if $w=x_{1} x_{2} \cdots x_{k} \in\left(A_{n}\right)^{k}$ (writing $k=k_{n}$ ), Proposition 5 shows that $\rho^{\prime}(w, e)=\sum \rho\left(x_{i}, e\right) \geqq 1$, and so $\rho^{\prime}(e, B) \geqq 1$ and $e \notin \bar{B}$, which proves the lemma.

With $\left\{A_{n}\right\}, A$ and $B$ as in the lemma, suppose that $A$ is not in $X$, and that $e \in \bar{A} \backslash A$. We shall show that $B$ is not closed in $\tau_{2}$. For if $\rho$ is any continuous pseudometric on $X$, and $\varepsilon>0$, there is an $x$ in some $A_{n}$ such that $\rho(x, e)<\varepsilon$. Setting $w(\alpha)=\alpha^{k}\left(k=k_{n}\right)$, we see that $w(x)=x^{k}$ and $w(e)=e$, so that

$$
\rho^{2}\left(x^{k}, e\right) \leqq \rho(x, e)<\varepsilon \text {. }
$$

Then since $x^{k} \in B, e$ is in the closure of $B$ in $\tau_{2}$. Thus, in the case when $e \in \bar{A} \backslash A, \tau_{1} \neq \tau_{2}$. An appeal to Proposition 7 now proves Case 2 in general.

Case 3: $\tau_{2}$ and $\tau_{3}$. We shall show that $\tau_{2} \neq \tau_{3}$ in the case $X=$ $[0,1]$. Let $I=[a, b] \subset[0,1]$ be a closed interval of length $1 / 4$, such that $\rho(I, e) \geqq 1 / 4$, where $\rho$ is the usual metric on $[0,1]$. For $n \in N$ and $1 \leqq l \leqq 3 n$, set $x_{l}^{n}=a+l / 12 n \in I$, define

$$
w_{n k}=x_{3 k-2}^{n}\left(x_{3 k-1}^{n}\right)^{-1} x_{3 k}^{n}\left(x_{2 k-2}^{n}\right)^{-1} x_{2 k-1}^{n}\left(x_{3 k}^{n}\right)^{-1} \quad \text { for } 1 \leqq k \leqq n,
$$

and set $w_{n}=w_{n 1} w_{n 2} \cdots w_{n n}$. We shall show that $\left\{w_{n}\right\}$ converges to $e$ in $\tau_{3}$, but does not in $\tau_{2}$. In general the superscripts $n$ will be omitted.

Let $S=\left\{s_{1}, \cdots, s_{m}\right\}$ be a finite ordered subset of $X$ containing $e$, define $G(S), d_{S}, d_{S}^{*}$ as earlier, and let $\phi: X \rightarrow \bigcup_{j=1}^{m-1} P\left(i\left(s_{j}\right), i\left(s_{j+1}\right)\right)$ be any continuous map such that $\phi\left(s_{j}\right)=i\left({ }_{j} s\right), j=1, \cdots, m$. Extend $\phi$ to a homomorphism $\Phi: F(X) \rightarrow G(S)^{*}$. Since $\phi$ is uniformly continuous on $X$, there is an $n_{0}$ such that when $n>n_{0}$, 


$$
\left\{\begin{aligned}
& d_{S}^{*}\left(\phi\left(x_{3 k-2}\right), \phi\left(x_{3 k-1}\right)\right)<1 \\
& d_{S}^{*}\left(\phi\left(x_{3 k-1}\right), \phi\left(x_{3 k}\right)\right)<1 \\
\text { and } \quad & d_{S}^{*}\left(\phi\left(x_{3 k-2}\right), \phi\left(x_{3 k}\right)\right)<1
\end{aligned}\right.
$$

for $1 \leqq k \leqq n$. We shall show that for each $t \in[0,1)$, at least two of $\phi\left(x_{3 k-2}\right)(t), \phi\left(x_{3 k-1}\right)(t)$ and $\phi\left(x_{3 k}\right)(t)$ are equal.

Consider a fixed value of $k$. Then for $l=0,1,2$ there is a $p_{l}$, $1 \leqq p_{l} \leqq m$, and an $\alpha_{l}, 0 \leqq \alpha_{l}<1$, such that

$$
\phi\left(x_{3 k-l}\right)(t)^{\prime}= \begin{cases}s_{p_{l}+1}, & 0 \leqq t<\alpha_{l} \\ s_{p_{l}}, & \alpha_{l} \leqq t<1 .\end{cases}
$$

For distinct $l_{1}$ and $l_{2}$, we must have $\left|p_{l_{1}}-p_{l_{2}}\right| \leqq 1$, since otherwise $\phi\left(x_{3 k-l_{1}}\right)(t)$ and $\phi\left(x_{3 k-l_{2}}\right)(t)$ are different for all $t \in[0,1)$, and hence $d_{S}^{*}\left(\phi\left(x_{3 k-l_{1}}\right), \phi\left(x_{3 k-l_{2}}\right)\right)=1$, contradicting (1). Therefore, two of $\left\{p_{0}, p_{1}, p_{2}\right\}$ are equal, and the third differs from them by at most one. Suppose without loss of generality that $p_{0}=p_{1}$, so that either (a) $p_{2}=p_{0}-1$, or (b) $p_{2}=p_{0}$, or (c) $p_{2}=p_{0}+1$.

Consider case (a). By (1), we must have $\alpha_{2}>\alpha_{0}$ and $\alpha_{2}>\alpha_{1}$. Then, assuming without loss of generality that $\alpha_{0} \leqq \alpha_{1}$, we have

$$
\begin{aligned}
\phi\left(x_{3 k}\right)(t) & =\phi\left(x_{3 k-1}\right)(t)=s_{p_{0}+1}, & & 0 \leqq t<\alpha_{0}, \\
\phi\left(x_{3 k}\right)(t) & =\phi\left(x_{3 k-2}\right)(t)=s_{p_{0}}, & & \alpha_{0} \leqq t<\alpha_{1}, \\
\text { and } \quad \phi\left(x_{3 k}\right)(t) & =\phi\left(x_{3 k-1}\right)(t)=s_{p_{0}}, & & \alpha_{1} \leqq t<1 .
\end{aligned}
$$

A similar analysis in the other cases shows that two of $\phi\left(x_{3 k-2}\right)(t)$, $\phi\left(x_{3 k-1}\right)(t)$ and $\phi\left(x_{3 k}\right)(t)$ are always equal, so that $\Phi\left(w_{n k}\right)(t)=e$ for all $t \in[0,1)$. Therefore $\Phi\left(w_{n}\right)(t)=e$ for all $t$ and for $n>n_{0}$, and hence $\rho_{S, \phi}\left(w_{n}, e\right)=0$ for $n>n_{0}$, and $w_{n} \rightarrow e$ with respect to $\rho_{S, \phi}$. Thus $w_{n} \rightarrow e$ in $\tau_{3}$.

To prove that $w_{n} \not f e$ in $\tau_{2}$, we use the corollary to Proposition 6 to give us, for a fixed $n$, representations $w\left(a_{1}, \cdots, a_{p}\right)=w_{n}$ and $w\left(b_{1}, \cdots, b_{p}\right)=e$, where $a_{1}, \cdots, a_{p}, b_{1}, \cdots, b_{p}$ are taken from among $e$ and $\left\{x_{l}^{n}: 1 \leqq l \leqq 3 n\right\}$, and where $\rho^{2}\left(w_{n}, e\right)=\sum_{i=1}^{p} \rho\left(a_{i}, b_{i}\right)$, with $\rho$ the usual metric on $[0,1]$. For a given $k, 1 \leqq k \leqq n$, consider all pairs $\left(a_{i}, b_{i}\right)$ for which $\left\{a_{i}, b_{i}\right\} \cap\left\{x_{3 k-2}, x_{3 k-1}, x_{3 k}\right\} \neq \phi$.

A straightforward argument shows that for (at least) one such pair we must have $a_{i} \neq b_{i}$. Write the pair as $(x(k), y(k))$, and carry out this procedure for each $k$. If any $x(k)$ or $y(k)=e$, we have $\rho^{2}\left(w_{n}, e\right) \geqq 1 / 4$ immediately, by our choice of the interval $I$. If no $x(k)$ or $y(k)=e$, then $\rho(x(k), y(k)) \geqq 1 / 12 n, 1 \leqq k \leqq n$. But the number of distinct pairs in $\{(x(k), y(k)): 1 \leqq k \leqq n\}$ is at least $n / 2$, as any such pair may equal at most one other. Therefore $\rho^{2}\left(w_{n}, e\right) \geqq$ $(n / 2) \cdot(1 / 12 n)=(1 / 24)$, and $w_{n} \neq e$ in $\tau_{2}$.

This completes the proof of Theorem 2 . 
EXAMPLe. Let $X$ be any uncountable set. Fixing $e \in X$, define a topology on $X$ by specifying that a set is closed if it is countable, or it contains $e$. Then $X$ is a (Hausdorff) normal nondiscrete space with the property that countable unions of closed sets are always closed. Thus Theorem 2 leaves the question of equality between $\tau_{0}$ and $\tau_{1}$, and $\tau_{1}$ and $\tau_{2}$, unsettled for a nontrivial class of spaces.

REMARK. We conclude by noting that if $X$ is a "large" metric space then $\tau_{3} \neq \tau_{1}$.

More precisely let $X$ be any metric space, or even any completely regular Hausdorff space which admits a continuous metric. Then Thompson [11] shows that $F(X)$ with the topology $\tau_{1}$ has no small subgroups. On the other hand, it is clear that if the cardinal number of the set $X$ is strictly greater than $2^{c}$, then from the definition, the group $F(X)$ with the topology $\tau_{3}$ does have small subgroups (because the cardinality of $G(S)^{*}$ is only $2^{c}$ ). Hence $\tau_{3} \neq \tau_{1}$ in this case.

\section{REFERENCES}

1. K. Bicknell and S. A. Morris, Norms on free topological groups, Bull. London Math. Soc., 10 (1978), 280-284.

2. R. Brown and S. A. Morris, Embeddings in contractible or compact objects, Colloq. Math., 38 (1978), 213-222.

3. T. H. Fay and B. V. Smith-Thomas, Free topological groups are almost never locally invariant, preprint.

4. M. I. Graev, Free topological groups, Izv. Akad. Nauk. SSSR. Ser. Mat., 12 (1948), 279-324 (Russian); English translation in Amer. Math. Soc. Transl., 35 (1951); reprinted in Amer. Math. Soc. Transl., 8 (1962), 305-364.

5. M. S. Khan, S. A. Morris and P. Nickolas, Local invariance of free topological groups, to appear.

6. S. A. Morris and Peter Nickolas, The extension of norms on subgroups of free topological groups, Proc. Amer. Math. Soc., 80 (1980), 185-188.

7. S. A. Morris and H. B. Thompson, Invariant metrics on free topological groups, Bull. Austral. Math. Soc., 9 (1973), 83-88.

8. S. Świerczkowski, Topologies in free algebras, Proc. London Math. Soc., (3) 14 (1964), 566-576.

9. W. Taylor, Varieties obeying homotopy laws, Canad. J. Math., 29 (1977), 498-527.

10. B. V. S. Thomas, Free topological groups, Gen. Top. and Appl., 4 (1974), 51-72.

11. H. B. Thompson, Remarks on free topological groups with no small subgroups, J. Anstral. Math. Soc., 18 (1974), 482-484.

Received March 6, 1981. Some of the work for this paper was done while the first author was a Visiting Professor at Tulane University, New Orleans.

La Trobe University

BUNDOORA, VIC. 3083

AUstralia

AND

UNIVERSITY OF QUEENSLAND

St. LuCIA, QLD., 4067

Australia 



\title{
PACIFIC JOURNAL OF MATHEMATICS
}

\section{EDITORS}

\author{
DONALD BABBITT (Managing Editor) \\ University of California \\ Los Angeles, CA 90024 \\ Hugo Rossi \\ University of Utah \\ Salt Lake City, UT 84112 \\ C. C. MOORE and ARthur Agus \\ University of California \\ Berkeley, CA 94720
}

J. DUGUNDJI

Department of Mathematics

University of Southern California

Los Angeles, CA 90007

R. FINN and J. MILGRAM

Stanford University

Stanford, CA 94305

\section{ASSOCIATE EDITORS}
R. ARENS
E. F. BeCKENBACH
B. H. NeumanN
F. WoLF
K. YoSHIDA

\section{SUPPORTING INSTITUTIONS}

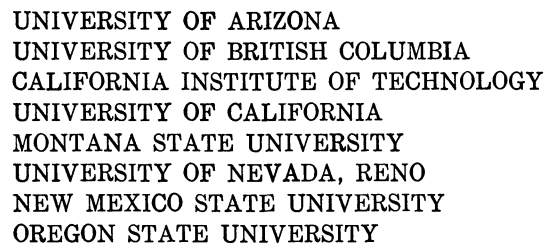

UNIVERSITY OF ARIZONA

UNIVERSITY OF BRITISH COLUMBIA

CALIFORNIA INSTITUTE OF TECHNOLOGY

UNIVERSITY OF CALIFORNIA

MONTANA STATE UNIVERSITY

UNIVERSITY OF NEVADA, RENO

NEW MEXICO STATE UNIVERSITY

OREGON STATE UNIVERSITY

\author{
UNIVERSITY OF OREGON \\ UNIVERSITY OF SOUTHERN CALIFORNIA \\ STANFORD UNIVERSITY \\ UNIVERSITY OF AAWAII \\ UNIVERSITY OF TOKYO \\ UNIVERSITY OF UTAH \\ WASHINGTON STATE UNIVERSITY \\ UNIVERSITY OF WASHINGTON
}

The Supporting Institutions listed above contribute to the cost of publication of this Journal, but they are not owners or publishers and have no responsibility for its content or policies,

Mathematical parers intended for publication in the Pacific Journal of Mathematics should be in typed form or offset-reproduced, (not dittoed), double spaced with large margins. Please do not use built up fractions in the text of the manuscript. However, you may use them in the displayed equations. Underline Greek letters in red, German in green, and script in blue. The first paragraph or two must be capable of being used separately as a synopsis of the entire paper. Please propose a heading for the odd unmbered pages of less than 35 characters. Manuscripts, in triplicate, may be sent to any one of the editors. Please classify according to the scheme of Math. Reviews, Index to Vol. 39. Supply name and address of author to whom proofs should be sent. All other communications should be addressed to the managing editor, or Elaine Barth, University of California, Los Angeles, California, 90024.

50 reprints to each author are provided free for each article, only if page charges have been substantially paid. Additional copies may be obtained at cost in multiples of 50 .

The Pacific Journal of Mathematics is issued monthly as of January 1966, Regular subscription rate: $\$ 114.00$ a year (6 Vol., 12 issues). Special rate: $\$ 57.00$ a year to individual members of supporting institution.

Subscriptions, orders for numbers issued in the last three calendar years, and changes of address shoud be sent to Pacific Journal of Mathematics, P.O. Box 969, Carmel Valley, CA 93924, U.S.A. Old back numbers obtainable from Kraus Periodicals Co., Route 100, Millwood, NY 10546.

PUBLISHED BY PACIFIC JOURNAL OF MATHEMATICS, A NON-PROFIT CORPORATION

Printed at Kokusai Bunken Insatsusha (International Academic Printing Co., Ltd.). 8-8, 3-chome, Takadanobaba, Shinjuku-ku, Tokyo 160, Japan. 


\section{Pacific Journal of Mathematics}

\section{Vol. 103, No. $2 \quad$ April, 1982}

Alberto Alesina and Leonede De Michele, A dichotomy for a class of positive

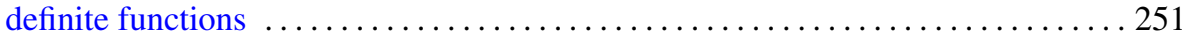

Kahtan Alzubaidy, Rank 2 -groups, $p>3$, and Chern classes . . . . . . . . . . 259

James Arney and Edward A. Bender, Random mappings with constraints on

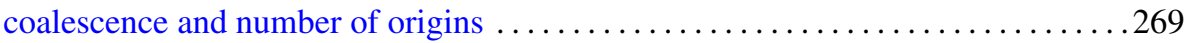

Bruce C. Berndt, An arithmetic Poisson formula . . . . . . . . . . . . . . . 295

Julius Rubin Blum and J. I. Reich, Pointwise ergodic theorems in 1.c.a. groups . . . 301

Jonathan Borwein, A note on $\varepsilon$-subgradients and maximal monotonicity . . . . . . . 307

Andrew Michael Brunner, Edward James Mayland, Jr. and Jonathan Simon,

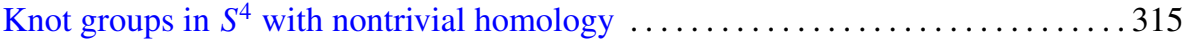

Luis A. Caffarelli, Avner Friedman and Alessandro Torelli, The two-obstacle

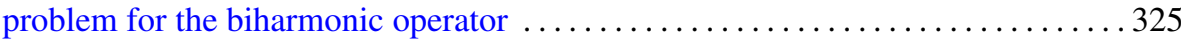

Aleksander Całka, On local isometries of finitely compact metric spaces . . . . . . 337

William S. Cohn, Carleson measures for functions orthogonal to invariant

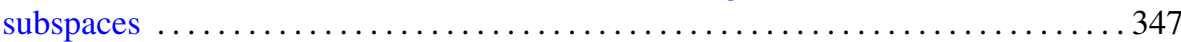

Roger Fenn and Denis Karmen Sjerve, Duality and cohomology for one-relator

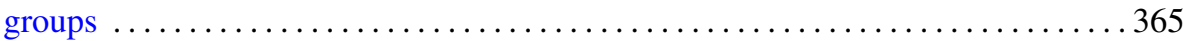

Gen Hua Shi, On the least number of fixed points for infinite complexes . . . . . . . 377

George Golightly, Shadow and inverse-shadow inner products for a class of linear

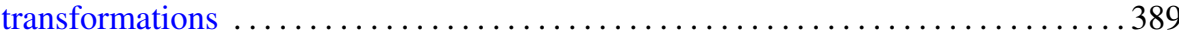

Joachim Georg Hartung, An extension of Sion's minimax theorem with an

application to a method for constrained games $\ldots \ldots \ldots \ldots \ldots \ldots \ldots \ldots \ldots \ldots . \ldots 4$

Vikram Jha and Michael Joseph Kallaher, On the Lorimer-Rahilly and

Johnson-Walker translation planes

Kenneth Richard Johnson, Unitary analogs of generalized Ramanujan sums .

Peter Dexter Johnson, Jr. and R. N. Mohapatra, Best possible results in a class of inequalities

Dieter Jungnickel and Sharad S. Sane, On extensions of nets

Johan Henricus Bernardus Kemperman and Morris Skibinsky, On the

characterization of an interesting property of the arcsin distribution ...

Karl Andrew Kosler, On hereditary rings and Noetherian $V$-rings

William A. Lampe, Congruence lattices of algebras of fixed similarity type. II . . . . 475

M. N. Mishra, N. N. Nayak and Swadeenananda Pattanayak, Strong result for real zeros of random polynomials

Sidney Allen Morris and Peter Robert Nickolas, Locally invariant topologies on free groups

Richard Cole Penney, A Fourier transform theorem on nilmanifolds and nil-theta functions

Andrei Shkalikov, Estimates of meromorphic functions and summability theorems

László Székelyhidi, Note on exponential polynomials

William Thomas Watkins, Homeomorphic classification of certain inverse limit spaces with open bonding maps $\ldots \ldots \ldots \ldots \ldots \ldots \ldots \ldots$

David G. Wright, Countable decompositions of $E^{n}$

Takayuki Kawada, Correction to: "Sample functions of Pólya processes" .

Z. A. Chanturia, Errata: "On the absolute convergence of Fourier series of the 